\title{
A compact CRLH metamaterial with wide band negative index characteristics
}

\author{
HARBINDER SINGH ${ }^{1, *}$, BALWINDER SINGH SOHI ${ }^{\mathbf{1}}$ and AMIT GUPTA ${ }^{2}$ \\ ${ }^{1}$ Department of ECE, Chandigarh University, Mohali, India \\ ${ }^{2}$ Department of ECE, I K Gujral Punjab Technical University, Jalandhar, India \\ *Author for correspondence (harbinder.ece@ cumail.in)
}

MS received 6 November 2018; accepted 1 February 2019; published online 3 June 2019

\begin{abstract}
Metamaterials are the artificial materials that may have a negative refractive index and results in extraordinary properties which are not found in nature. This research focusses on designing a compact-sized artificial material for negative characteristics of permittivity, permeability and refractive index over the wide range of frequencies. The metamaterial design is evaluated for single negative, double negative and double positive behaviours over a broad range of frequencies to investigate the gap and gapless transitions from backward to forward wave propagation. The dispersion diagram of the material is also investigated for left or right hand behaviour of the material. The equivalent circuit diagrams along with circuit simulations of the parameters are also presented for detailed analysis and understanding. The designed metamaterial behaves like a double negative material for low frequencies and double positive material for high frequencies. The approach is verified using CST microwave studio simulation and the results obtained are validated using the rectangular waveguide measurement method.
\end{abstract}

Keywords. CRLH; DNG; left hand material; NRW; SNG.

\section{Introduction}

The electromagnetic behaviour of any material can be depicted with the help of two basic parameters i.e., permittivity $(\varepsilon)$ and permeability $(\mu)$. The refractive index is also one of the important parameters of a material, which further depends on the permittivity and permeability and decides the amount of refraction and reflection in any material. Metamaterials are the artificial material that may have either negative permittivity or permeability or both may be negative simultaneously [1]. Depending on the signs of these two parameters (permittivity and permeability), the material can be classified into four categories [2]. All the natural materials have positive values of permittivity and permeability and are classified as double positive (DPS) material. Single negative (SNG) materials are also possible with a single negative value of permittivity or permeability [3]. These single negative metamaterials include epsilon negative (ENG) and mue negative (MNG) materials [4]. If the permittivity of the material is negative with positive permeability, then they are known as ENG materials. On the other hand, if the permeability of the material is negative with positive permittivity, then they are known as MNG materials. The last type of metamaterial is double negative (DNG) materials, which are having negative values of permittivity and permeability [5]. DNG metamaterials are not found in nature and have a negative refractive index, which result in extraordinary properties. Some of them include negative refraction, backward wave propagation, inverse Doppler effect, perfect lens, cloaking and many more [6,7].

The term 'metamaterial' was first coined by Victor Veselago [8] in his theory. He has pointed out that the wave propagation through such a material will be in the opposite direction than the naturally occurring materials. The concept was converted into reality by Smith et al [9] by simultaneously verifying the negative values of permeability and permittivity. The split ring resonators (SRR) were designed for this purpose, which result in a left handed materials and follow a reverse Doppler effect, backward wave propagation and inverse Snell's law. Since the Smith experiment prepared the DNG materials and confirmed the Veselago's theory about the negative refractive index, the study of left handed materials has become a new frontier that attracts scientific community's attention.

There are various metamaterial designs available in the literature which result in metamaterial behaviours like diamondshaped [10], cross-shaped [11], concentric ring resonators [12], EM resonators [13], n-point star resonators [14], bed nails [15], fishnet [16], modified S-shaped SRR [17], complementary quad spiral resonator [18] and many more for different types of metamaterial applications. However, most of the reported metamaterials are found either having the SNG or narrow band DNG responses. Moreover, most of the designs required the vias through the substrate and are complex in design with immense unit cell dimensions. The fabrication and placement of these bulky 
designs are difficult for most of the metamaterial applications.

This paper aims to discuss the step by step process of metamaterial designing for wide band negative index applications and is categorized into five sections. The study is initialized with the introduction, to give a brief idea about the concept and literature. Section 2 presents the detailed discussion of metamaterial designing using a circuit modelling approach. The detailed analysis of a parameter retrieval technique and the required simulation setup are described in section 3 . The microstrip implementation and result analysis along with experimental verification are described in section 4. Eventually, the study is concluded in section 5 .

\section{Circuit modelling of a CRLH metamaterial}

One of the best methods to design and analyse the metamaterial is transmission line theory, as it is capable of expressing the complex properties of metamaterial in terms of circuit elements [19]. The theory is based on right hand ( $\mathrm{RH})$ and left hand ( $\mathrm{LH})$ transmission lines depending on the RH and LH behaviours of the wave propagation. The RH transmission lines are commonly used in real life, but the LH transmission lines are not found easily because of the non-availability of natural LH materials. However, the artificial materials can be realized to mimic the $\mathrm{LH}$ behaviour effectively and can be analysed by composite right left hand (CRLH) transmission line [20]. The equivalent circuit model of loss less CRLH is illustrated in figure 1 (the losses are ignored to simplify the model).

The series inductance $\left(L_{\mathrm{R}}\right)$ and shunt capacitance $\left(C_{\mathrm{R}}\right)$ contribute to the RH and LH behaviours are due to series capacitance $\left(C_{\mathrm{L}}\right)$ and shunt inductance $\left(L_{\mathrm{L}}\right)$. The transmission line will behave as a pure right hand $(\mathrm{PRH})$, if the $\mathrm{LH}$ components have infinite values i.e., $L_{\mathrm{L}}=C_{\mathrm{L}}=\infty$ resulting in RH immittance only. On the other hand, the transmission line will behave as pure left hand (PLH), if the RH components have zero values i.e., $L_{\mathrm{R}}=C_{\mathrm{R}}=0$ resulting in LH immittance only [21]. However, the existence of the PLH transmission line is not possible in real life due to parasitic

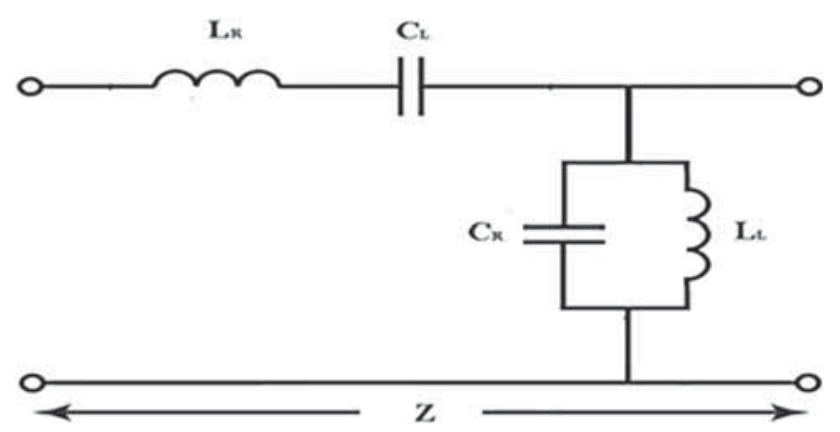

Figure 1. Equivalent circuit model for the CRLH transmission line. inductance and capacitance effects in series and shunt, respectively. So practically, $L_{\mathrm{R}}$ and $C_{\mathrm{R}}$ cannot be zero on the account of parasitic effects of metal, especially at high frequencies. The CRLH is the combination of PRH and PLH, thus serving as a good approximation of metamaterials [22]. Such a type of metamaterial can be designed with the knowledge of circuit element values. This further requires the mathematical dependency of these values on frequencies [23], so that the material properties for left and right can be engineered. The resonating frequencies for $\mathrm{RH}$ and $\mathrm{LH}$ can be represented as:

$$
\begin{aligned}
& f_{\mathrm{R}}=\frac{1}{2 \pi \sqrt{L_{\mathrm{R}} C_{\mathrm{R}}}}, \\
& f_{\mathrm{L}}=\frac{1}{2 \pi \sqrt{L_{\mathrm{L}} C_{\mathrm{L}}}} .
\end{aligned}
$$

Similarly, the series and shunt resonance frequencies for the transmission line can be expressed as:

$$
\begin{aligned}
& f_{\mathrm{se}}=\frac{1}{2 \pi \sqrt{L_{\mathrm{R}} C_{\mathrm{L}}}}, \\
& f_{\mathrm{sh}}=\frac{1}{2 \pi \sqrt{L_{\mathrm{L}} C_{\mathrm{R}}}} .
\end{aligned}
$$

The line will support forward or backward wave propagations, depending on the transition from the $\mathrm{LH}$ and $\mathrm{RH}$ frequency ranges. The transition frequency for the CRLH transmission line is given by:

$$
f_{\mathrm{o}}=\sqrt{f_{\mathrm{R}} f_{\mathrm{L}}}=\sqrt{f_{\mathrm{se}} f_{\mathrm{sh}}} .
$$

The right and left hand impedances can be expressed as:

$$
\begin{aligned}
& Z_{\mathrm{R}}=\sqrt{\frac{L_{\mathrm{R}}}{C_{\mathrm{R}}}}, \\
& Z_{\mathrm{L}}=\sqrt{\frac{L_{\mathrm{L}}}{C_{\mathrm{L}}}} .
\end{aligned}
$$

The left and right hand behaviours can be analysed by plotting the dispersion diagram for these CRLH lines [24]. This requires the calculation of propagation constant $(\gamma)$, attenuation constant $(\alpha)$ and phase constant $(\beta)$ over a broad range of operating frequency $(f)$.

$$
\begin{aligned}
& \gamma=\alpha+j \beta, \\
& \gamma=j s(f) \sqrt{\left(\frac{f}{f_{\mathrm{R}}}\right)^{2}+\left(\frac{f_{\mathrm{L}}}{f}\right)^{2}-\left(\frac{L_{\mathrm{R}}}{L_{\mathrm{L}}}+\frac{C_{\mathrm{R}}}{C_{\mathrm{L}}}\right)} .
\end{aligned}
$$

The real and imaginary parts of the propagation constant are known as the attenuation and phase constant, respectively. Thus, depending on the sign within the radical for equation (8), it may be purely imaginary or purely real (despite the loss less line consideration). Depending on this, the function $s(f)$ is defined as:

$$
s(f)=\left\{\begin{array}{l}
-1, \text { if } f<\min \left(f_{\mathrm{se}}, f_{\mathrm{sh}}\right) \text { LH range } \\
+1, \text { if } f>\max \left(f_{\mathrm{se}}, f_{\mathrm{sh}}\right) \text { RH range }
\end{array} .\right.
$$


The metamaterial parameters like relative permittivity $\left(\varepsilon_{\mathrm{r}}\right)$, permeability $\left(\mu_{\mathrm{r}}\right)$ and refractive index $(n)$ can also be calculated in terms of circuit elements as a function of frequency [25] and are given by:

$$
\begin{aligned}
& \varepsilon=\varepsilon_{\mathrm{r}} \varepsilon_{\mathrm{o}}=C_{\mathrm{R}}-\frac{1}{(2 \pi f)^{2} L_{\mathrm{L}}}, \\
& \mu=\mu_{\mathrm{r}} \mu_{\mathrm{o}}=L_{\mathrm{R}}-\frac{1}{(2 \pi f)^{2} C_{\mathrm{L}}}, \\
& n=\sqrt{\mu_{\mathrm{r}} \varepsilon_{\mathrm{r}}}=c \frac{\beta}{2 \pi f} .
\end{aligned}
$$

Depending on the designing requirement of metamaterial application, the values of circuit elements can be calculated from these equations. In this research, the transition frequency targeted is $6.8 \mathrm{GHz}$. Thus, the metamaterial will transit from LH to RH behaviour around this frequency. One of the possible calculated circuit element values for this transition are $L_{\mathrm{R}}=0.1885 \mathrm{nH}, C_{\mathrm{R}}=1.2396 \mathrm{pF}, L_{\mathrm{L}}=0.4170 \mathrm{nH}$ and $C_{\mathrm{L}}=3.1806 \mathrm{pF}$. The metamaterial is analysed with circuit simulation for these values.

The dispersion diagram and refractive index for these circuit values are illustrated in figures 2 and 3, respectively, which shows that the material is supporting backward waves $<6.5 \mathrm{GHz}$ and forward waves $>7 \mathrm{GHz}$. Thus, in a low frequency range $<6.5 \mathrm{GHz}$, which is the series resonating frequency $\left(f_{\mathrm{se}}\right)$ of the circuit and is given by equation (3), the LH immittance dominates and the material will behave as the LH material. On the other hand, in a high frequency range $>7 \mathrm{GHz}$, which is the shunt resonating frequency $\left(f_{\mathrm{sh}}\right)$ of the circuit and is given by equation (4), the RH immittance dominates and the material will behave as the RH material. However, the transition from LH to RH propagation is separated by a gap of $0.5 \mathrm{GHz}$, which acts as a forbidden band for propagation. The forbidden band is in between $f_{\text {se }}$ and $f_{\text {sh }}$ in which only attenuation exists. In general, this is because, the series resonance is not equal to shunt resonance, and is

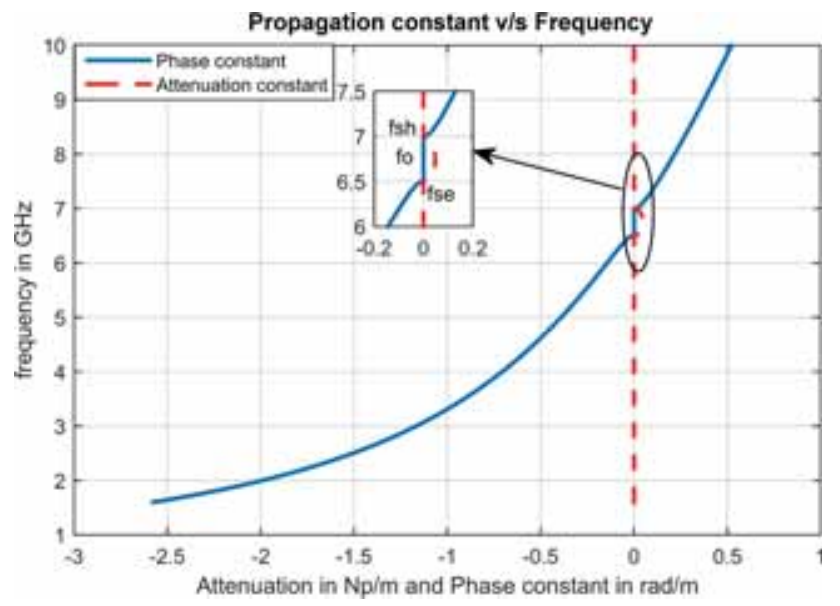

Figure 2. Dispersion diagram for the unbalanced case.

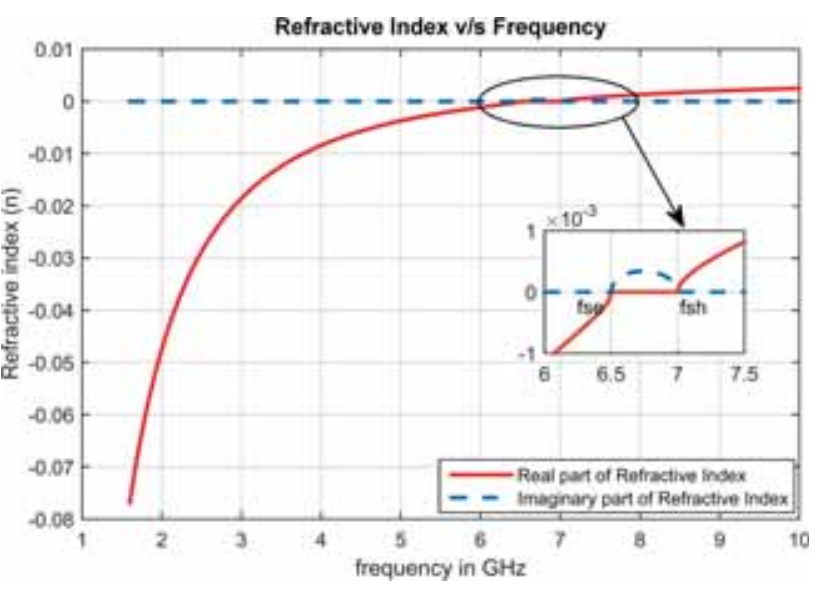

Figure 3. Refractive index for the unbalanced case.

known as the unbalanced CRLH case. If the series and shunt resonating frequencies are the same, then the transition from the LH to RH region will be gapless and result in a balanced CRLH material [26]. This can be achieved by ensuring the balanced condition, given by equation (12).

$$
\begin{aligned}
& f_{\mathrm{se}}=f_{\mathrm{sh}}, \\
& L_{\mathrm{R}} C_{\mathrm{L}}=L_{\mathrm{L}} C_{\mathrm{R}} .
\end{aligned}
$$

Thus, in the case of unbalanced CRLH, the transition from LH to RH will not be gapless and as a result of this, the forbidden band comes into existence between $f_{\text {se }}$ and $f_{\text {sh. }}$. Within this forbidden gap, the permittivity and permeability will behave differently and can be analysed by equations (9 and 10). The $\mu$ will be negative for frequencies $<f_{\mathrm{se}}$ and $\varepsilon$ will be negative $<f_{\text {sh }}$, making only one out of two to be negative in a gap and results in SNG behaviour, that can be either MNG or ENG behaviour. The refractive index plot in figure 3 illustrates that the real part of the refractive index is negative $<f_{\mathrm{se}}$ and is positive $>f_{\mathrm{sh}}$ and is zero within the forbidden gap. Thus, the material behaves as the DNG material $<f_{\text {se }}$ and the DPS material $>f_{\mathrm{sh}}$ and as the ENG material within the forbidden gap.

To design a balanced CRLH metamaterial, the first thing to ensure is the balanced condition or alternatively the impedance matching. The right and left hand impedance given by equations ( 6 and 7), respectively, should be matched properly with the characteristic impedance $\left(Z_{0}\right)$. Hence, the series and shunt frequencies will be equivalent to each other and results in gapless transition from the LH to RH range. Keeping these conditions in mind, the values of circuit elements are recalculated for the transition frequency of $6.8 \mathrm{GHz}$ and are given as: $L_{\mathrm{R}}=0.2261 \mathrm{nH}, C_{\mathrm{R}}=1.3281 \mathrm{pF}$, $L_{\mathrm{L}}=0.4124 \mathrm{nH}$ and $C_{\mathrm{L}}=2.4218 \mathrm{pF}$.

The dispersion diagram and refractive index for the balanced CRLH case are illustrated in figures 4 and 5, respectively, which shows that the material is supporting backward waves $<6.8 \mathrm{GHz}$ and forward waves $>6.8 \mathrm{GHz}$. Thus, the 


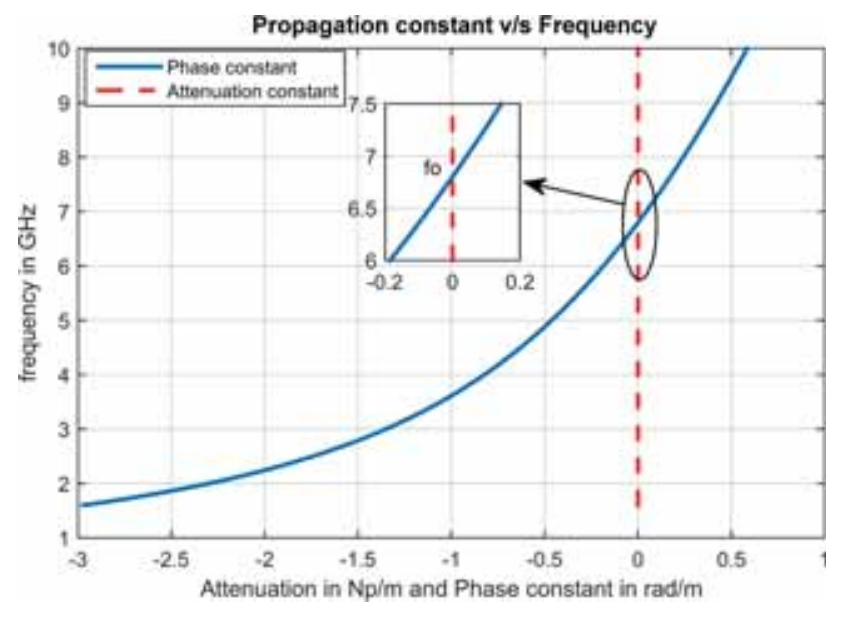

Figure 4. Dispersion diagram for the balanced case.

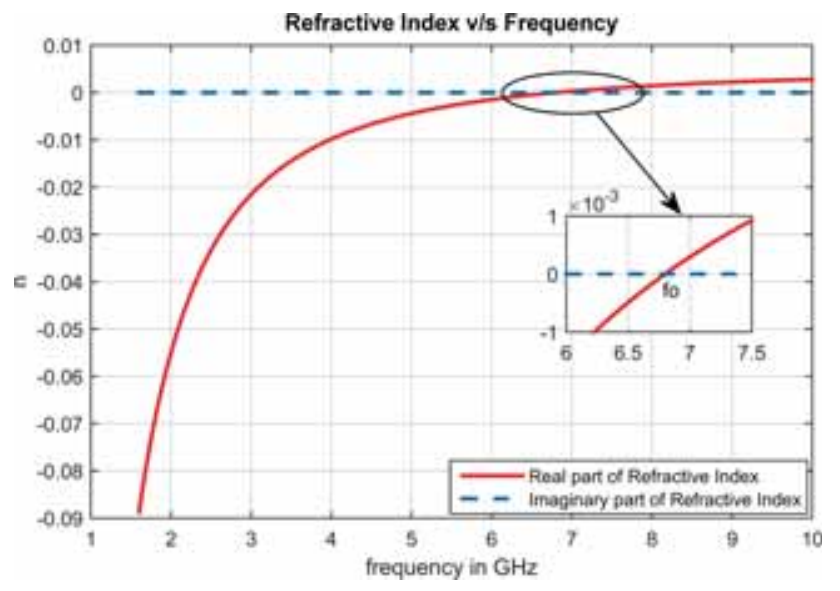

Figure 5. Refractive index for the balanced case.

transition from the $\mathrm{LH}$ to $\mathrm{RH}$ range is gapless and occurs at a transaction frequency of $6.8 \mathrm{GHz}$ with no forbidden band. This is because of the proper impedance matching condition, which results in the series and shunt resonance frequencies to be equal to the transition frequency. The refractive index plot for the balanced case in figure 5 illustrates that the real part of the refractive index is negative below $f_{\mathrm{o}}$ and is positive above $f_{\mathrm{o}}$ with no forbidden gap. Thus, the material behaves as the DNG material below $f_{\mathrm{o}}$ and the DPS material above $f_{\mathrm{o}}$. The imaginary part of the refractive index is zero, but this is valid for the loss less case only [27]. In the case of losses, which can be represented by series resistance and shunt admittance in equivalent circuit, the refractive index becomes complex and the imaginary parts may be non-zero [28].

The circuit simulation results obtained for the CRLH metamaterial have to be fabricated practically by engendering the calculated component values $\left(L_{\mathrm{R}}=0.2261 \mathrm{nH}, C_{\mathrm{R}}=\right.$ $1.3281 \mathrm{pF}, L_{\mathrm{L}}=0.4124 \mathrm{nH}$ and $C_{\mathrm{L}}=2.4218 \mathrm{pF}$ ). The implementation can be possible with the use of microstrip line technology for implementing an inductor and capacitance equivalent $[29,30]$. Once the equivalent is designed, the parameters (refractive index, permittivity and permeability) have to be retrieved for verification of LH and RH behaviours over the broad range of frequency [31]. The simulation setup required and the retrieval techniques used for the unambiguous determination of parameters are discussed in section 3 .

\section{Parameter retrieval and simulation setup}

Metamaterial properties like refractive index, intrinsic impedance, loss tangent, etc. can be calculated with the help of permittivity and permeability. The complex values of these parameters can be retrieved using various parameter retrieval techniques and these techniques further depend on the measurement methods. The transmission-reflection technique is one of the famous retrieval techniques for metamaterials and is capable of retrieving the values of permittivity and permeability with the help of the reflection and transmission coefficient measurement method [32]. This method is mainly suitable for structures from which reflection and transmission parameters can be measured with the help of waveguides [33]. NicolsonRoss-Weir (NRW) [34,35] is one of the famous techniques for retrieval of complex values of permittivity and permeability from reflection and transmission coefficient measurement, but it suffers from branching phase ambiguity [36,37].

The NRW approach is based on the transmission/reflection line method in which the parameters are retrieved by measuring $S_{11}$ and $S_{21}$. This approach can be applied to a metamaterial slab of the known thickness $d$ for the retrieval of unknown permittivity and permeability. As the wave propagates through the material, some of the incident waves will reflect back to the source and rest of the wave will transmit through the thickness $d$ of the slab as shown in figure 6 .

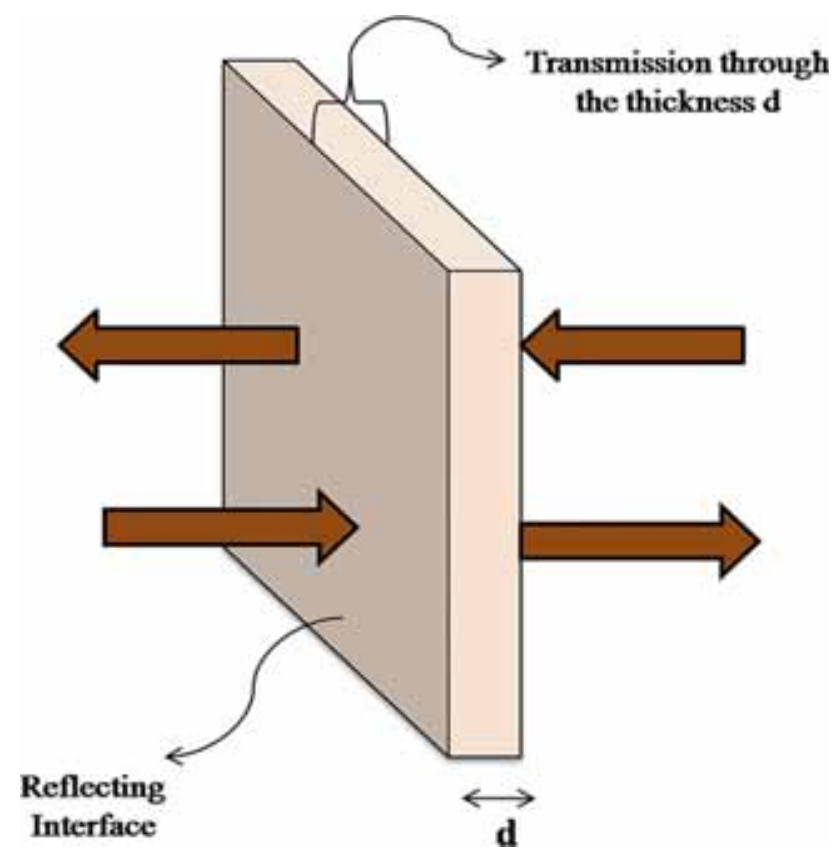

Figure 6. Illustration of reflecting and transmitting interfaces. 
The summations of all the reflected and transmitted waves are the total reflection and transmission respectively and can be represented mathematically as:

$$
\begin{aligned}
& S_{11}=\frac{\left(1-t^{2}\right) r}{1-r^{2} t^{2}}, \\
& S_{21}=\frac{\left(1-r^{2}\right) t}{1-r^{2} t^{2}},
\end{aligned}
$$

where $r$ and $t$ are the reflection and transmission coefficients and are given by:

$$
\begin{aligned}
& r=\frac{\eta-\eta_{0}}{\eta+\eta_{0}}, \\
& t=e^{-j k_{0} n d},
\end{aligned}
$$

where $\eta$ and $\eta_{0}$ are the intrinsic impedance of the slab and free space, respectively. $k_{0}$ and $n$ are the phase constant and refractive index of the slab, respectively.

NRW suggests the finding of reflection coefficient from scattering parameters using an intermediate parameter $X$ and the intrinsic impedance is retrieved from the calculated reflection coefficient. Alternatively, the intrinsic impedance can also be derived directly in terms of scattering coefficients [36] using equations (13-15):

$$
\eta= \pm \sqrt{\frac{\left(1+S_{11}\right)^{2}-S_{21}^{2}}{\left(1-S_{11}\right)^{2}-S_{21}^{2}}} .
$$

The intrinsic impedance expressed in equation (17) has a sign ambiguity. The solution to this problem is to choose a sign for the passive material i.e., $\operatorname{Re}(\eta) \geq 0$. The reflection coefficient can be obtained from the calculated intrinsic impedance using equation (15). The transmission coefficient can also be obtained in terms of the reflection coefficient using equation (18):

$$
t=\frac{S_{21}}{1-S_{11} r} .
$$

The main limitation of NRW is the ambiguity in the refractive index $n$ because of the branching problem of an algorithm. The correct expression of the refractive index can be obtained from equation (16) and is given by equation (19):

$$
\begin{aligned}
& \ln \left(e^{-j k_{0} n d}\right)=-j\left(k_{0} n d+2 \pi m\right), \\
& \ln (t)=-j\left(k_{0} n d+2 \pi m\right), \\
& n=\frac{j \ln (t)-2 \pi m}{k_{0} d}
\end{aligned}
$$

where branching variable $m$ is an unknown integer value from $-\infty \leq m \leq \infty$. One of the best approaches to tackle with the branching problem of NRW is suggested by Chen et al [38],

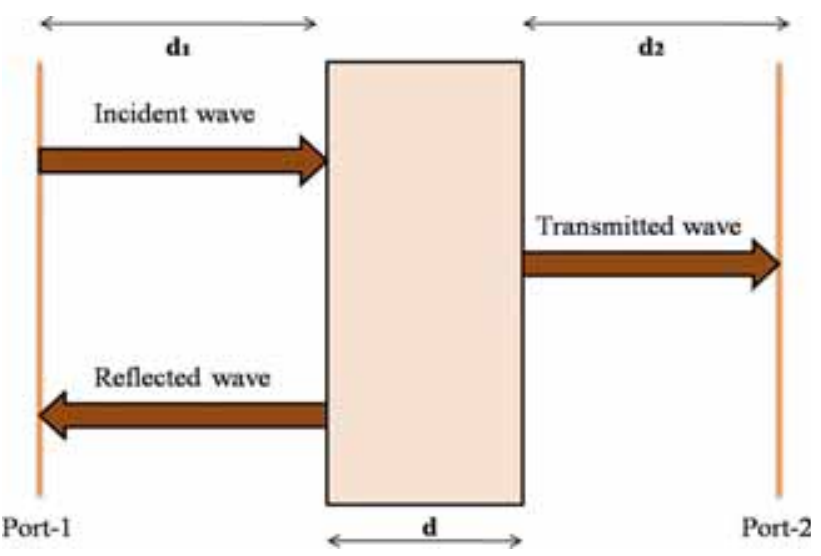

Figure 7. Simulation equivalent model.

they suggest a method of finding out the branching variable $m$ with the help of binomial function.

Equations (17 and 19) represent the derived expression of the intrinsic impedance and refractive index of the slab. However, the general expressions of them in terms of permittivity and permeability are given in equations (20 and 21):

$$
\begin{aligned}
& \eta=\eta_{0} \sqrt{\frac{\mu_{\mathrm{r}}}{\epsilon_{\mathrm{r}}}}, \\
& n=\sqrt{\mu_{\mathrm{r}} \epsilon_{\mathrm{r}}} .
\end{aligned}
$$

The ultimate aim of this retrieval approach is to retrieve the permittivity and permeability of the material. The same can be obtained by multiplying and dividing equations (20 and 21) to obtain the expression of $\mu_{\mathrm{r}}$ and $\epsilon_{\mathrm{r}}$.

$$
\begin{aligned}
\mu_{\mathrm{r}} & =n \frac{\eta}{\eta_{0}}, \\
\epsilon_{\mathrm{r}} & =n \frac{\eta_{0}}{\eta},
\end{aligned}
$$

where $n$ and $\eta$ can be retrieved from equations (17 and 19). Now, the effective permittivity and permeability can be retrieved with the help of the intrinsic impedance and unambiguous refractive index using equations (22 and 23). The scattering parameters required for retrieval can be simulated using a full wave electromagnetic simulator. The CST microwave studio is used as the full wave electromagnetic simulator in this study, and the simulation equivalent of figure 6 is represented in figure 7.

In a simulation model, two exciting ports are used to simulate scattering parameters $S_{11}$ and $S_{21}$ or $S_{22}$ and $S_{12}$. The exciting ports are at a distance of $d_{1}$ and $d_{2}$ from the material of the known thickness $d$. The boundary conditions $[39,40]$ are specified considering the waveguide equivalent model. 


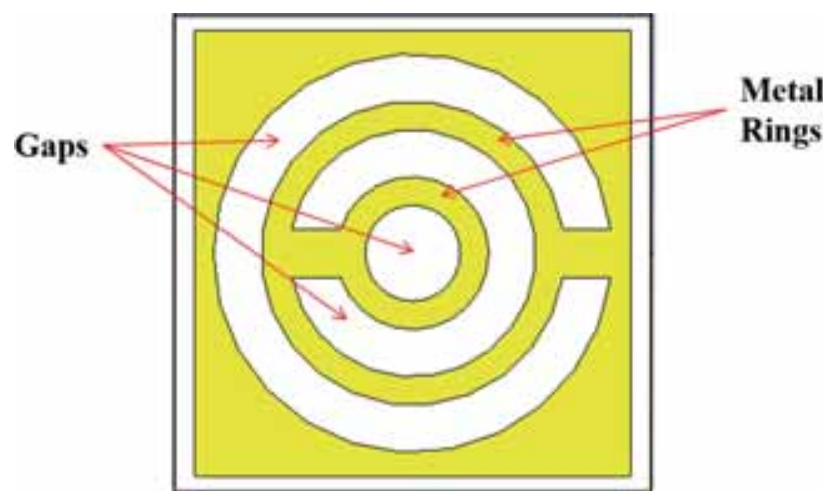

Figure 8. Illustration of capacitance and inductance effects.

\section{Microstrip implementation and result analysis}

The CRLH metamaterial described in section 2 is implemented with the use of microstrip technology. The motive of the microstrip designing is to implement the inductor and capacitance equivalent of figure 1 . The values of the inductance are intentionally chosen to be low to avoid the special requirements of vias through the substrate. The requirement of the via free structure can be achieved by initializing the design with the famous complementary split ring resonators (CSRR) [41]. The modified-CSRR structure for a small-sized optimized unit cell is shown in figure 8, which consists of a square-shaped $\left(5 \times 5 \mathrm{~mm}^{2}\right)$ single-side printed metallic structure on the FR4 (lossy) substrate. The dielectric constant of the substrate is 4.3 with a thickness of $1.574 \mathrm{~mm}$ and a loss tangent of 0.025 . The metallic material used is copper with electric conductivity $(\sigma)$ of $5.8 \times 107 \mathrm{~S} \mathrm{~m}^{-1}$ and a thickness of $35 \mu \mathrm{m}$.

The structure will behave as an LC resonator, where the inductance effect is due to two metal rings equivalent to two turns of the inductance with different radii. The innermost circular gap is inserted intentionally to introduce the two-ring inductance effects unlike the case of the standard CSRR. The inductance can be controlled to limited values by changing the ring radius. On the other hand, the capacitance effect is approximated by the gaps between these rings and can be controlled by varying the gaps between these rings. These controlling parameters are optimized until the values of the equivalent lumped elements are tuned approximately to that of the desired transition frequency. The parameter retrieval technique discussed in section 3 is used to extract the complex parameters of this lossy CRLH unit cell.

The retrieved permittivity, permeability and refractive index for the structure of figure 8 are illustrated in figures 9-11, respectively, which shows that the permeability is negative for frequencies below $f_{\text {se }}$ and permittivity is negative below $f_{\mathrm{sh}}$. Thus, the material is supporting backward waves $<6.5 \mathrm{GHz}$ and forward waves $>7 \mathrm{GHz}$, but the transition from LH to RH propagation is separated by a gap of $0.5 \mathrm{GHz}$. The refractive index plot in figure 11 illustrates that

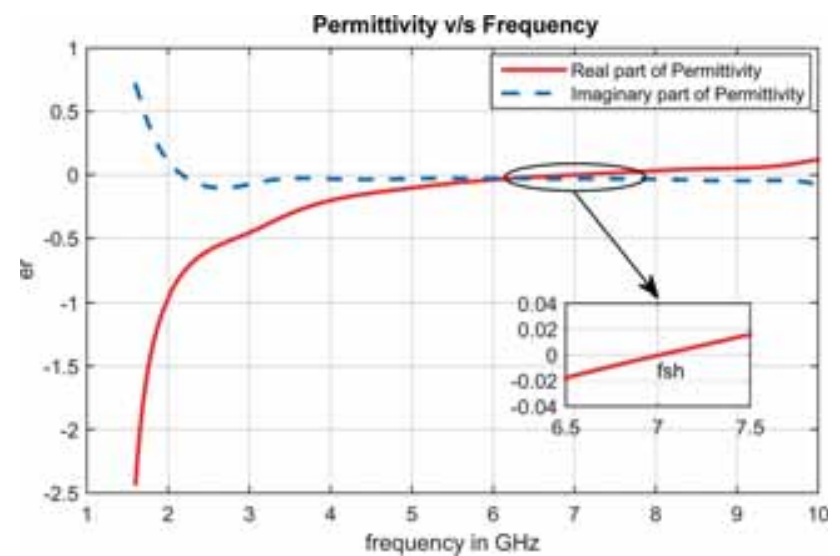

Figure 9. Retrieved permittivity for the unbalanced case.

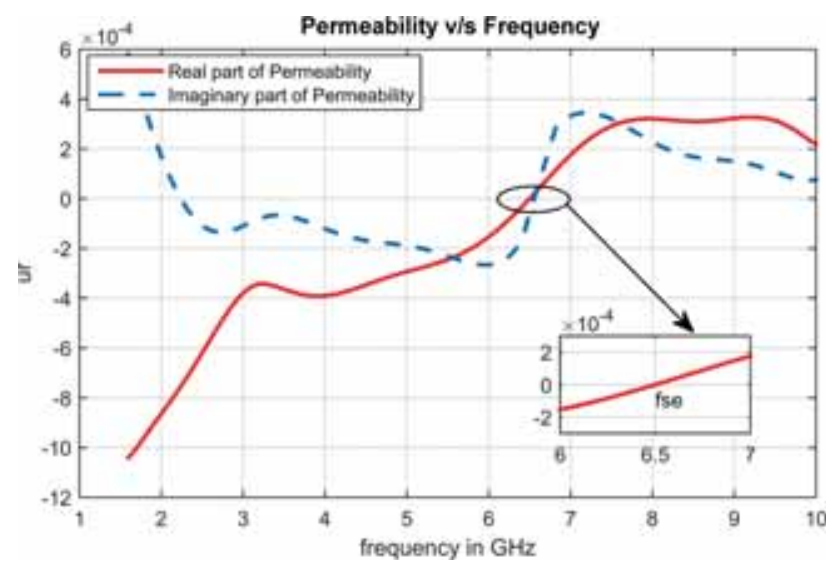

Figure 10. Retrieved permeability for the unbalanced case.

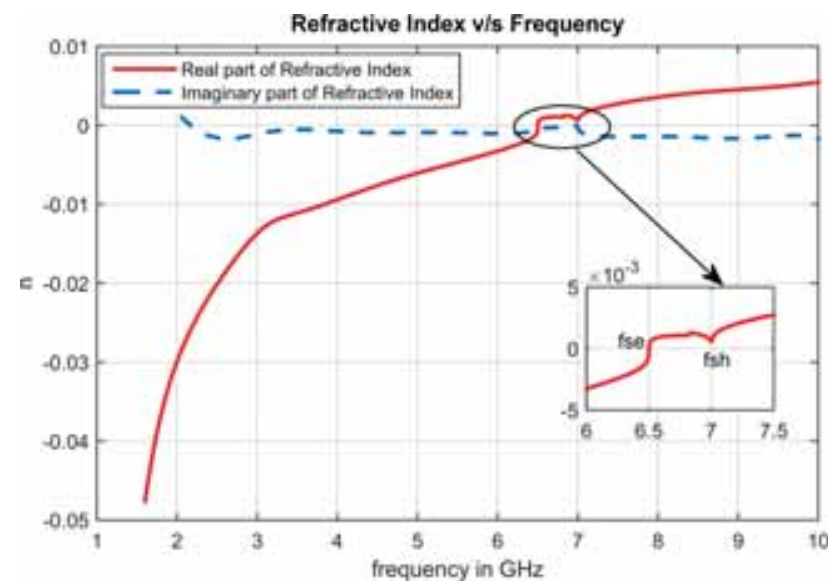

Figure 11. Retrieved refractive index for the unbalanced case.

the real part of the refractive index is negative below $f_{\text {se }}$ and is positive above $f_{\mathrm{sh}}$ and is near to zero within the transition gap. Thus, the material behaves as the DNG material below $f_{\text {se }}$ and the DPS material above $f_{\mathrm{sh}}$ and as ENG within the transition gap. In general, this transition gap is on account of unequal 


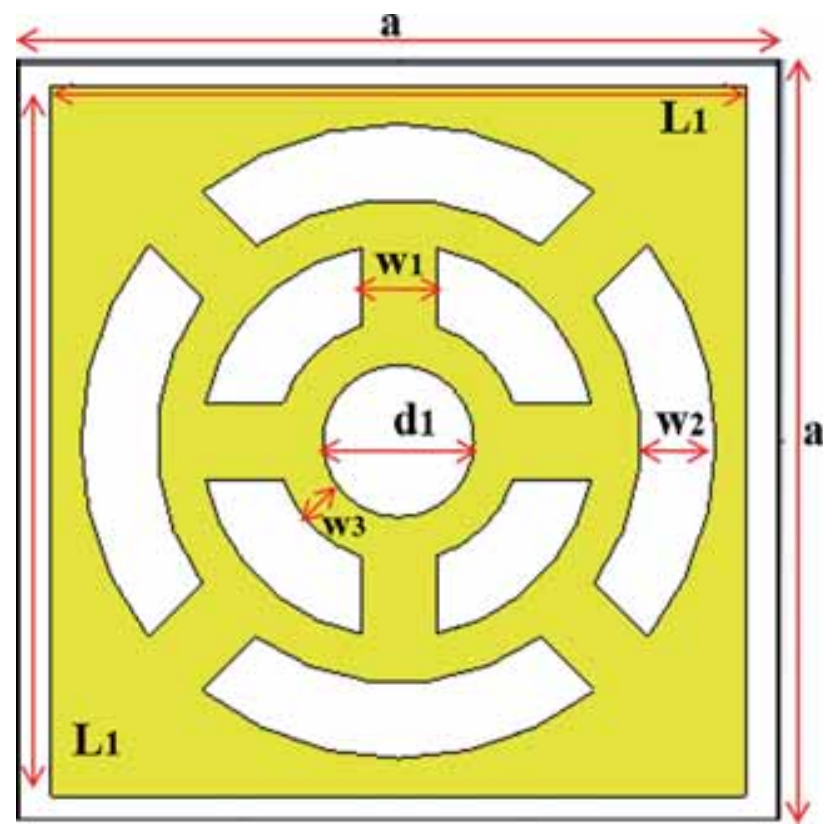

Figure 12. Balanced CRLH unit cell.

series and shunt resonance and is the unbalanced CRLH case.

A small transition gap can be covered by synthesizing the structure with the help of controlling parameters to achieve the balanced condition of CRLH. The LC resonance behaviours of the structure can be controlled further by insertion of the slits in rings. The introduction of slits results in decreased capacitance and increased inductance. The quad slit operation on the unbalanced structure of figure 8 is illustrated in figure 12. The transition from the $\mathrm{LH}$ to $\mathrm{RH}$ range can be controlled by controlling the metal ring radius, gaps and slits, which is equivalent to controlling the resonance effect of a resonator. The controlling parameters are optimized for different configurations until the structure is tuned to the balanced condition and refractive index obtained for some of the configurations is illustrated in figure 13. The transition gap from $\mathrm{LH}$ to $\mathrm{RH}$ for configurations 2 and 4 is $0.5 \mathrm{GHz}$ each, which is the same as that obtained for the structure as shown in figure 8 , but for different shunt and series frequencies. The refractive index of configuration 2 is near to zero from 6.3 to $6.8 \mathrm{GHz}$, while that of configuration 4 is from 6.8 to $7.3 \mathrm{GHz}$. On the other hand, the transition gap for configuration 3 is reduced to $0.3 \mathrm{GHz}$ with near to zero refractive index from 6.5 to $6.8 \mathrm{GHz}$, but results in an unbalanced case of the CRLH structure. The transition from LH to RH for configuration 1 is gapless at a transition frequency of $6.8 \mathrm{GHz}$ and hence, results in a balanced CRLH structure. The concluded structure and optimized parameters of the configuration 1 are shown in figure 12 and table 1 , respectively.

The retrieved permittivity, permeability and refractive index for the concluded structure of figure 12 are illustrated in figures 14-16, respectively. All the three retrieved parameters

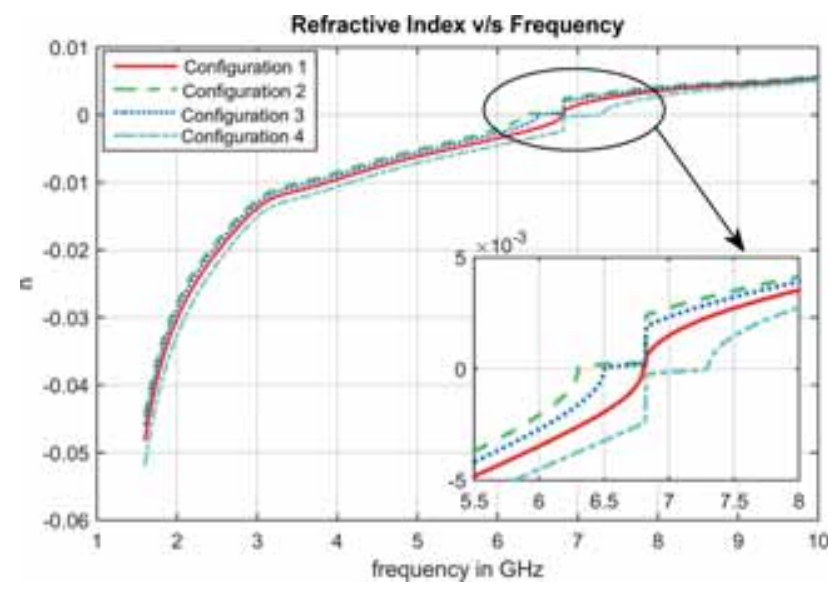

Figure 13. Refractive index for different configurations.

Table 1. Dimensions of optimized CRLH balanced unit cell.

\begin{tabular}{lc}
\hline Parameter & Dimension (mm) \\
\hline$a$ & 5 \\
$L 1$ & 4.6 \\
$d 1$ & 1 \\
$w 1$ & 0.5 \\
$w 2$ & 0.5 \\
$w 3$ & 0.3 \\
\hline
\end{tabular}

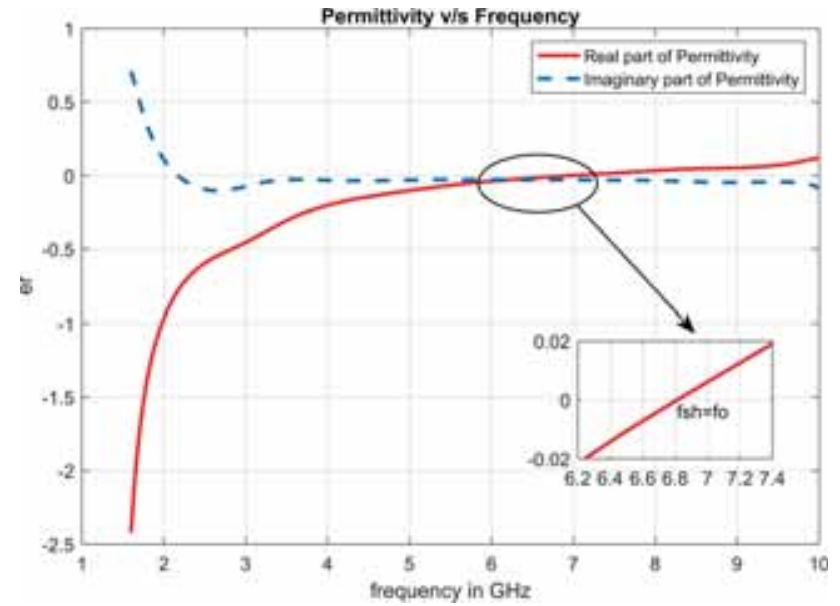

Figure 14. Retrieved permittivity for the balanced case.

are changing from negative to positive values at the same transition frequency $f_{\mathrm{o}}$, thus the structure is resonating at the same series and shunt frequency and results in a balanced CRLH case. Hence, the material is supporting backward waves $<6.8 \mathrm{GHz}$ and forward waves $>6.8 \mathrm{GHz}$ with a gapless transition from the DNG to DPS propagation range. The dispersion diagram in figure 17 illustrates that the material behaves as the LH material below $f_{\mathrm{o}}$ and the $\mathrm{RH}$ material above $f_{\mathrm{o}}$. In general, a balanced CRLH structure is obtained 


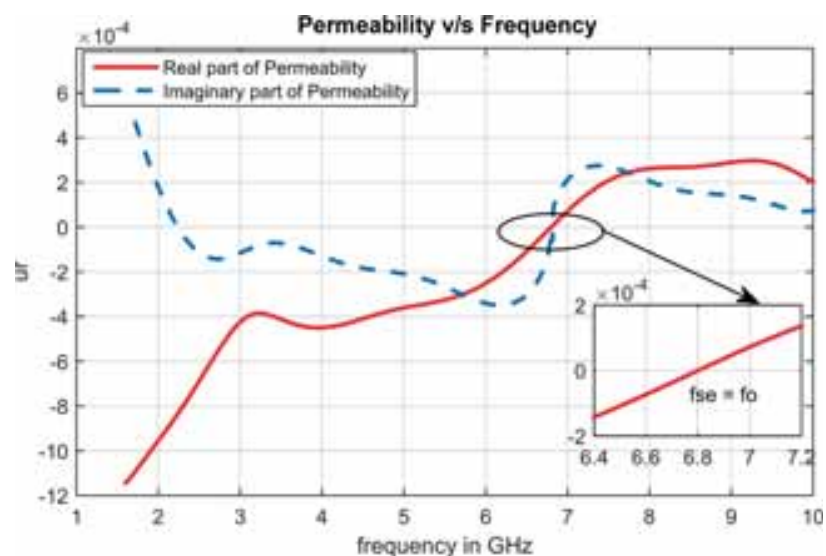

Figure 15. Retrieved permeability for the balanced case.

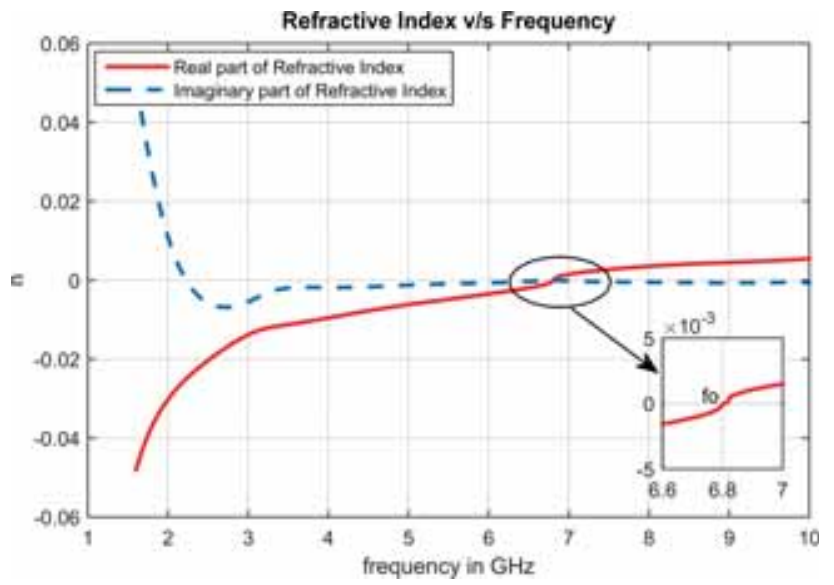

Figure 16. Retrieved refractive index for the balanced case.

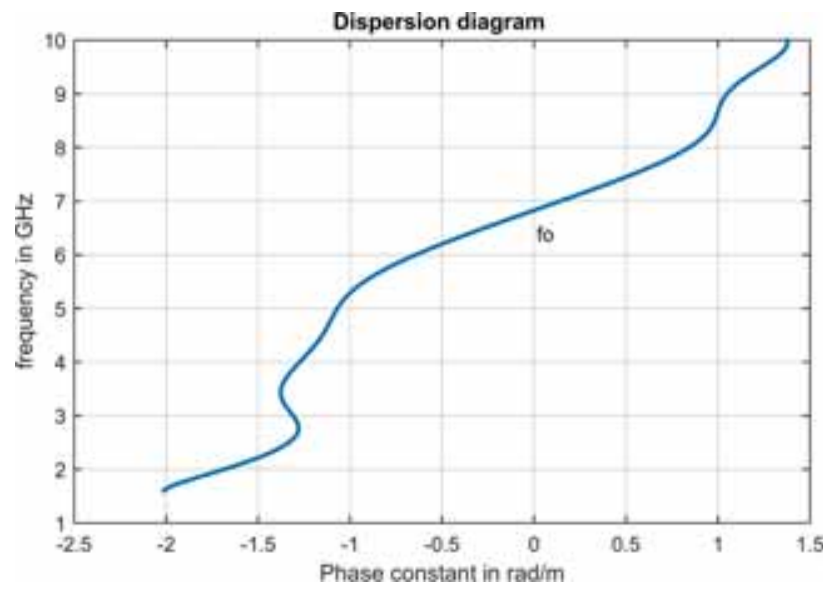

Figure 17. Retrieved dispersion diagram for the balanced case.

with wide negative index characteristics for a compact-sized unit cell.

The simulation results obtained for the negative index balanced structure can be verified with the help of scattering parameter measurement using a vector network analyser

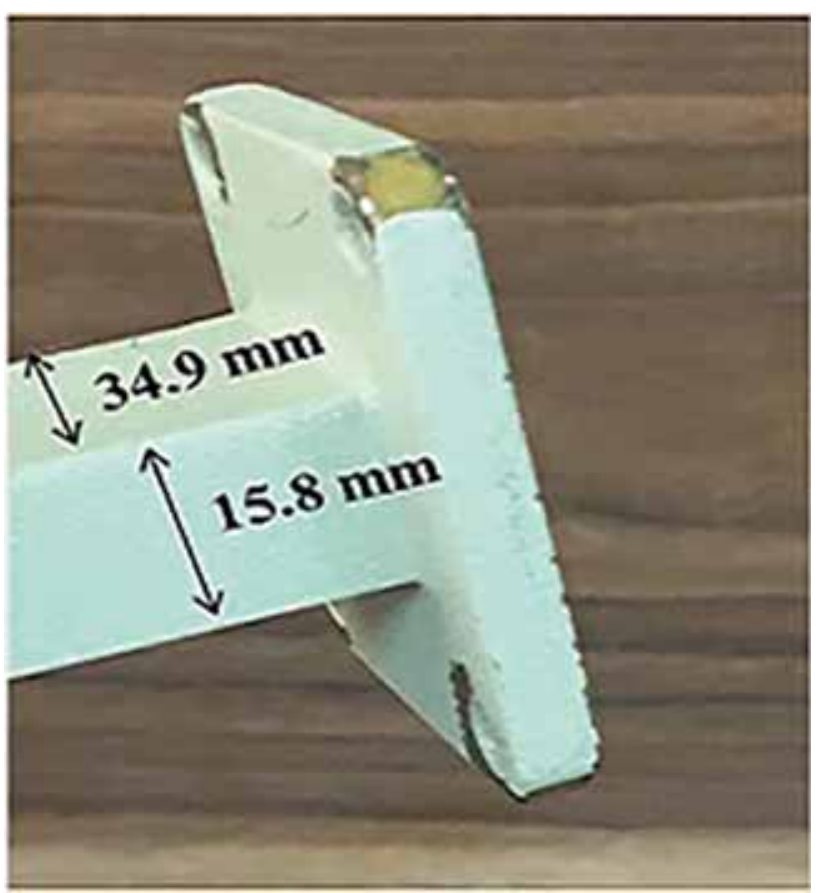

Figure 18. WR 137 rectangular waveguide.

(VNA). Most of the scattering measurement techniques required a sample holder $[42,43]$ and the special requirement of the sample holder can be avoided with the use of rectangular waveguide measurement. The dimensions of the waveguide will depend on the frequency range, in which parameters are to be retrieved for unknown materials. For experimental purpose, the material under test (MUT) is tested for $\mathrm{C}$ band frequencies, the motive of this range is to include the transition frequency $\left(f_{\mathrm{o}}=6.8 \mathrm{GHz}\right)$ in the testing range so that the DNG and DPS behaviours of the balanced structure can be verified. The waveguide standard for the $\mathrm{C}$ band (part) is WR 137 and the dimensions of this standard wave guide are $34.9 \times 15.8 \mathrm{~mm}^{2}$ as shown in figure 18 . The cut off frequency $\left(f_{\mathrm{c}}\right)$ for the lowest order mode of WR 137 is $4.301 \mathrm{GHz}$. Mono-mode operation can be ensured by using the frequency range in between $5.85-8.2 \mathrm{GHz}$ [44]. Thus, the frequency range to be used in measurement should be in between 5.85 and $8.2 \mathrm{GHz}$.

The scattering parameters are measured using VNA (model no. MS46322A) at the two ports of the waveguides with the MUT placed in between the cross-sections of the waveguide as shown in figure 19. The MUT needs to be prepared for proper fitting within the waveguide, the prototype array of $7 \times 3$ unit cells of the proposed balanced CRLH unit cell is fabricated considering the homogeneous properties [21,45] and is shown in figure 20. However, in simulation, the MUT is considered as a two port network as illustrated in figure 2 . Thus, they need to be calibrated according to figure 2 with the help of through reflect line (TRL) calibration [46]. In TRL, the two waveguides (WR 137) are connected directly 


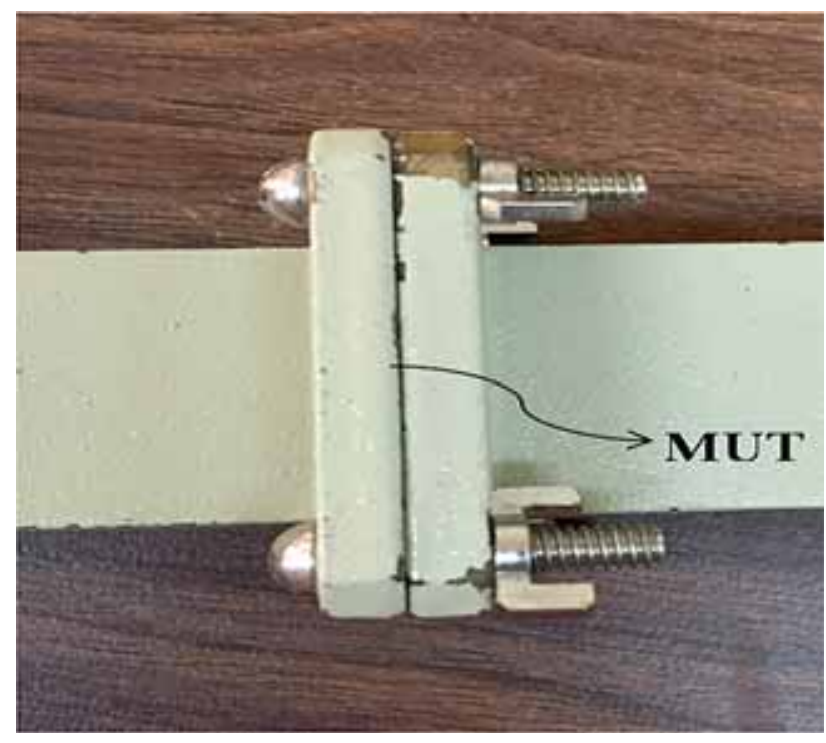

Figure 19. Illustration of MUT placed between the waveguides.

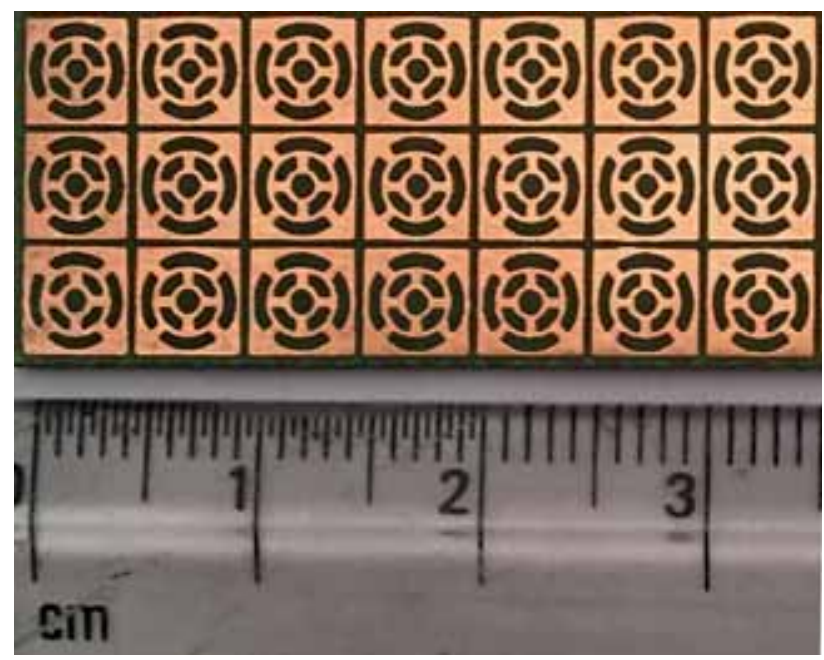

Figure 20. Fabricated prototype.

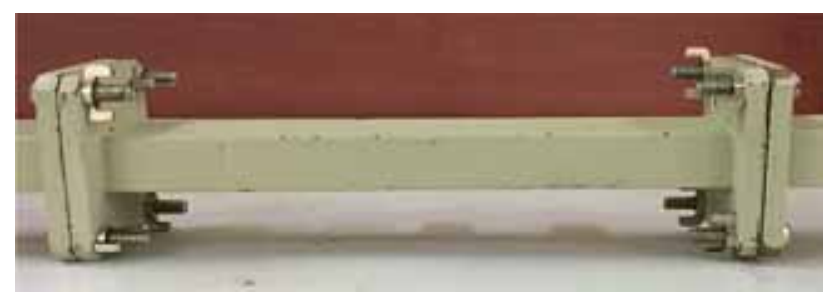

Figure 21. TRL calibration of waveguide.

to each other (through) and scattering parameters $S_{11}$ and $S_{21}$ are obtained, then, the second waveguide is replaced with a reflector plane (reflect) and $S_{11}$ is measured. Finally, a waveguide line of identical dimension (line) is connected between two waveguide adaptors and $S_{11}, S_{12}, S_{21}$ and $S_{22}$ are obtained as illustrated in figure 21. Now, with the use of TRL measurement and de-embedded function of VNA, the waveguides are

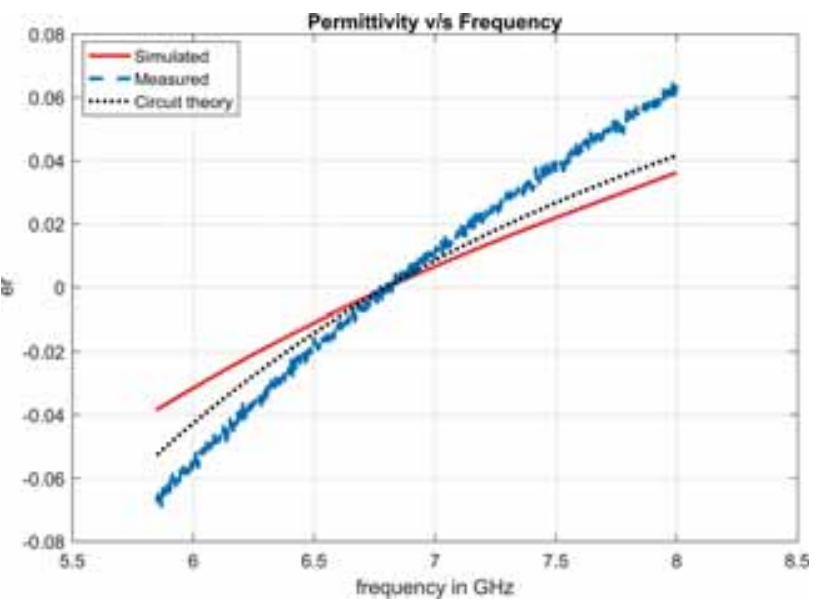

Figure 22. Measured permittivity for the balanced case.

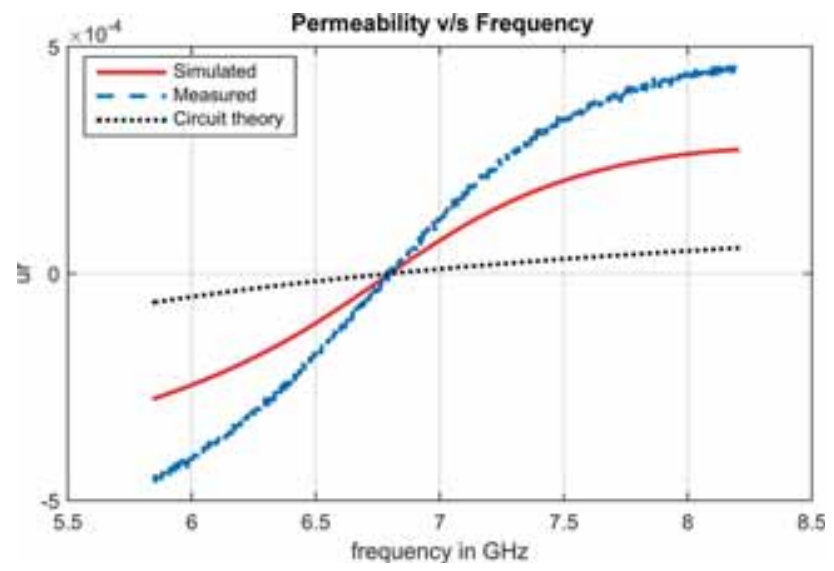

Figure 23. Measured permeability for the balanced case.

calibrated. Once the scattering parameters are measured for MUT, the parameters are retrieved according to the algorithm of section 2 .

The measured permittivity, permeability, refractive index and dispersion diagram for the concluded structure are illustrated in figures 22-25, respectively. The measured results are compared with that of simulation and circuit theory results. All the three results (measured, simulated and circuit theory) validate that the structure is shifting from $\mathrm{LH}$ to $\mathrm{RH}$ behaviour at the same transition frequency $f_{\mathrm{o}}$ of $6.8 \mathrm{GHz}$. Hence, the structure is behaving as a balanced CRLH metamaterial without any transition gap. Thus, a good agreement between the measured and simulated results is obtained. The measured permittivity and permeability plot is negative up to the same frequency of $6.8 \mathrm{GHz}$ and results in the same shunt and series resonating frequencies. Therefore, the metamaterial mimic as a DNG material up to $6.8 \mathrm{GHz}$ and the DPS material $>6.8 \mathrm{GHz}$. The backward behaviour of the metamaterial can also be analysed from the dispersion diagram and refractive index plots, which suggests that the metamaterial will support the backward wave $<6.8 \mathrm{GHz}$ and the forward wave 


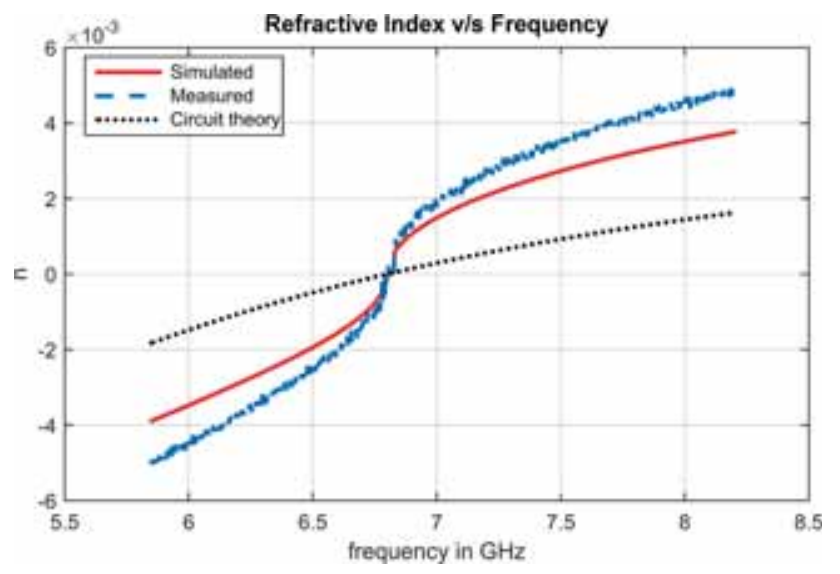

Figure 24. Measured refractive index for the balanced case.

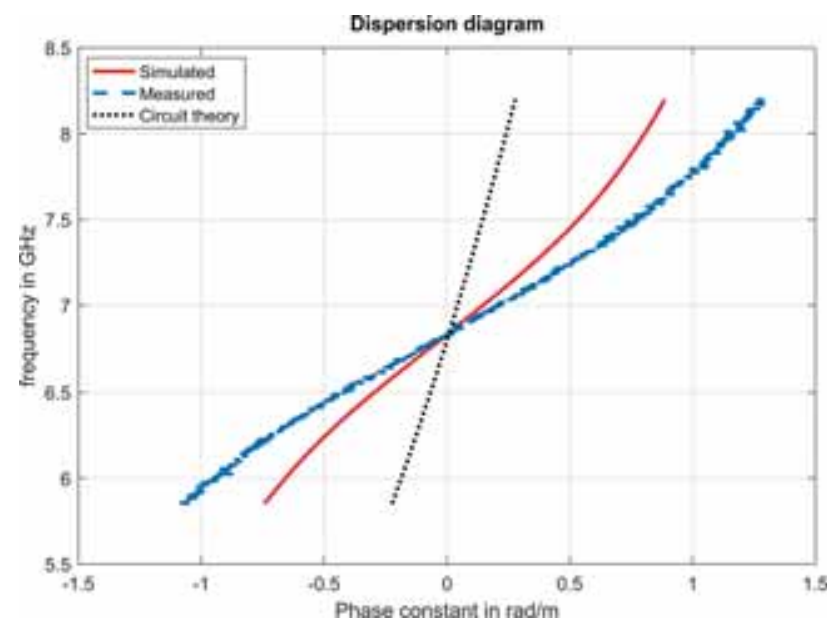

Figure 25. Measured dispersion diagram for the balanced case.

$>6.8 \mathrm{GHz}$. Hence, a compact balanced CRLH metamaterial with broadband negative index characteristics is obtained whose negative index characteristics can be engineered by scaling and managing the controlling parameters of the structure.

\section{Conclusion}

The study describes the detailed analysis of a novel balanced CRLH structure. The permittivity, permeability and refractive index obtained for the designed metamaterial are shifting from negative to positive values at the same transition frequency of $6.8 \mathrm{GHz}$, therefore, the structure is resonating at the same series and shunt frequency and results in a balanced CRLH case. Hence, the material is supporting backward waves $<6.8 \mathrm{GHz}$ and forward waves $>6.8 \mathrm{GHz}$ with a gapless transition from the DNG to DPS propagation range and can be scaled for other frequencies as well by engineering the controlling parameters. The dispersion diagram of the material is also investigated, which reveals that the metamaterial is behaving as a $\mathrm{LH}$ material $<6.8 \mathrm{GHz}$ and a $\mathrm{RH}$ material $>6.8 \mathrm{GHz}$. Thus, a balanced CRLH structure is obtained with wide negative index characteristics. The design is analysed and supported by circuit theory of CRLH metamaterials. The equivalent circuit diagrams along with circuit simulations of the parameters are also presented for detailed analysis and understanding. The approach is verified using CST microwave studio simulation, circuit simulation and the results obtained are validated with the use of the rectangular waveguide approach for the $\mathrm{C}$ band and a good approximation between them is obtained. Moreover, the designed unit cell is planar in structure and is free from the special requirement of the vias through the substrate. This single-side printed planar structure can be fabricated easily and is compact enough $\left(5 \times 5 \times 1.609 \mathrm{~mm}^{3}\right)$ to be fit easily in any metamaterial applications.

\section{References}

[1] Pendry J B 2000 Phys. Rev. Lett. 853966

[2] Rani R, Kaur P and Verma N 2015 Int. J. Hybrid. Inf. Technol. 8199

[3] Feng T, Han H, Wang L, Yang F and Zhang F 2018 J. Mater. Sci. Mater. Electron. 2911886

[4] Ferdous S, Hossain A, Mahmud S, Chowdhury H, Rahman M, Mahdy C et al 2013 IET Microw. Antennas Propag. 7768

[5] Carbonell J, Rogla L J, Boria V E and Lippens D 2006 IEEE Trans. Microw. Theory. Tech. 541527

[6] Markley L and Eleftheriades G V 2007 IEEE Antennas Wirel. Propag. Lett. 628

[7] Van T P, Park J W, Lam V D, Jang W H, Choi E H, Nikitov S A et al 2012 J. Light Technol. 303451

[8] Veselago V G 1968 Sov. Phys. USPEKHI 10509

[9] Smith D R, Padilla W J, Vier D C, Nemat-Nasser S C and Schultz S 2000 Phys. Rev. Lett. 844184

[10] Urbani F 2010 IEEE Antennas Wirel. Propag. Lett. 9720

[11] Amiri N, Forooraghi K and Atlasbaf Z 2011 IEEE Antennas Wirel. Propag. Lett. 10524

[12] Chen M, Meng D, Jiang H and Wang Y 2018 Shock Vib. 20181

[13] Karamanos T D, Dimitriadis A I and Kantartzis N V 2012 IEEE Antennas Wirel. Propag. Lett. 11480

[14] Kaur K P and Upadhyaya T 2018 IET Microw. Antennas Propag. 121428

[15] Polemi A, Maci S and Kildal P S 2011 IEEE Trans. Antennas Propag. 53904

[16] Mahmood A, Ogucu Yetkin G and Sabah C 2017 Adv. Condens. Matter Phys. 20171

[17] Mahmood A, Ögücü Yetkin G and Sabah C 2017 Adv. Condens. Matter Phys. 20171

[18] Sarkar A, Adhikary M, Sharma A, Biswas A, Akhtar M J and Hu Z 2018 IET Microw. Antennas Propag. Res. 121310

[19] Lai A, Caloz C and Itoh T 2004 IEEE Microw. Mag. 534

[20] Sanada A, Caloz C and Itoh T 2004 IEEE Microw. Wirel. Components Lett. 1468

[21] Caloz C and Itoh T 2004 IEEE Trans. Antennas Propag. 52 1159 
[22] Caloz C, Sanada A and Itoh T 2004 IEEE Trans. Microw. Theory Tech. $\mathbf{5 2} 980$

[23] Durán-Sindreu M, Naqui J, Selga J, Vélez P, Bonache J and Martín F 2013 Wiley Encycl. Electr. Electron. Eng. J. G. Webster 51

[24] Herraiz-Martínez F J, González-Posadas V, Iñigo-Villacorta F and Segovia-Vargas D 2007 IEEE Microw. Wirel. Components Lett. 1713

[25] Eleftheriades G V 2009 Mater. Today 1230

[26] Gil M, Bonache J, Selga J, García J and Martín F 2007 IEEE Microw. Wirel. Components Lett. 1797

[27] Tümkaya M A, Karaaslan M and Sabah C 2018 Bull. Mater. Sci. 4191

[28] Caloz C and Itoh T 2004 Microw. Opt. Technol. Lett. 43 112

[29] Caloz C and Itoh T 2002 IEEE Antennas Propag. Soc. Int. Symp. (IEEE Cat. No. 02CH37313) (San Antonio, TX, USA: IEEE) p 412

[30] Tong W, Hu Z, Zhang H, Caloz C and Rennings A 2008 IET Microw. Antennas Propag. 2731

[31] Caloz C, Chang C C and Itoh T 2001 J. Appl. Phys. 905483

[32] Singh H, Sohi B S and Gupta A 2018 J. Microw. Power Electromagn. Energy $\mathbf{5 2} 214$

[33] Dubrovskiy S and Gareev K 2015 Young Res. Electr. Electron. Eng. Conf. (EIConRusNW), IEEE, St. Petersbg., p 24
[34] Nicolson A M and Ross G F 1970 IEEE Trans. Instrum. Meas. 19377

[35] Weir W B 1974 Proc. IEEE 6233

[36] Qi J, Wang N and Xiao S 2017 IEEE Trans. Dielectr. Electr. Insul. 241852

[37] Hollander Y and Shavit R 2011 IET Microw. Antennas Propag. 584

[38] Chen X, Grzegorczyk T M, Wu B, Pacheco J and Kong J A 2004 Phys. Rev. E 701

[39] Szabó Z, Park G, Hedge R and Li E 2010 IEEE Trans. Microw. Theory Tech. $\mathbf{5 8} 2646$

[40] Cai T, Wang G and Liang J 2014 IEEE. Antennas Wirel. Propag. Lett. 13555

[41] Rao M V, Madhav B T P, Anilkumar T and Nadh B P 2018 AEU-Int. J. Electron. Commun. 97229

[42] Abadla M M, Taya S A and Shabat M M 2011 J. Nanoelectron. Optoelectron. 91823

[43] Hosseini M H, Heidar H and Shams M H 2017 IEEE Trans. Instrum. Meas. 66148

[44] Costa F, Monorchio A and Pietro V G 2011 IEEE Antennas Wirel. Propag. Lett. 1011

[45] Trang F, Rogalla H and Popovic Z 2015 IEEE Trans. Appl. Supercond. 251

[46] Hammler J, Gallant A J and Balocco C 2016 IEEE Trans. TeraHertz Sci. Technol. 6817 